\title{
A Soft X-ray Halo of the LMC?
}

\author{
M.J. Blondiau \\ Radioastronomisches Institut der Universität Bonn, D-53121 Bonn, Germany
}

\begin{abstract}
ROSAT X-ray observations of the LMC show several X-ray shadows in the $3 / 4 \mathrm{keV}$ and $1.5 \mathrm{keV}$ bands. The $1 / 4 \mathrm{keV}$ band is dominated by emission of the galactic foreground. It is uncertain whether there is additional soft X-ray contribution from the LMC. The so-called HI-L-component is located in front of the HI-D-component and therefore the matter of the L-component accounts for most of the observed X-ray shadows. The $1 / 4 \mathrm{keV}$ ROSAT image of the LMC reveals a slightly fainter $\mathrm{X}$-ray emission region at the position of the eastern part of the HI-L-component. This suggests the existence of a soft X-ray halo in the LMC. Investigations of the X-ray shadows show that also the lower energy end of the $3 / 4 \mathrm{keV}$ band originates partly from this soft X-ray plasma which is located in front of the absorption features. This additional soft X-ray emission, in the lower energy end of the $3 / 4 \mathrm{keV}$ band, is brighter than the typical diffuse galactic emission at the same galactic latitude.
\end{abstract}

\section{Introduction}

The investigation of X-ray shadows is a powerful tool to disentangle the 3D structure of the interstellar medium on large angular scales. Wang et al. (1991) verified the existence of a widely distributed X-ray gas in the LMC. These authors also discovered the first X-ray shadow in the LMC. The largest $\mathrm{X}$-ray shadow in the LMC is located south of the giant HII region 30 Doradus. In the ROSAT X-ray images, different spatial parts of this shadow show up in different energy bands. The dark western part shows up in the ROSAT $3 / 4 \mathrm{keV}$ and $1.5 \mathrm{keV}$ bands (Blondiau et al. 1997), while the easternmost part is detected in the softer $R O S A T 1 / 4 \mathrm{keV}$ band. In the $R O S A T 3 / 4 \mathrm{keV}$ and $1.5 \mathrm{keV}$ bands the structure of a $T=10^{6.7} \mathrm{~K}$ hot plasma in the LMC is visible (Blondiau 1996). The question is whether there is a contribution from a softer diffuse X-ray emitting plasma of the LMC also in the $1 / 4 \mathrm{keV}$ band.

\section{Data}

I have analyzed pointed X-ray data of the LMC extracted from the ROSAT public archive. The whole area of Fig. 2 is a mosaic of 172 individual pointed PSPC observations. The minimum integration time is $1 \mathrm{ksec}$ while the maximum is about $204 \mathrm{ksec}$ and is reached in the region of the supernova 1987A. The data are corrected for the various effects associated with the so-called non-cosmic X-ray backgrounds (for details see Blondiau et al. 1997). 

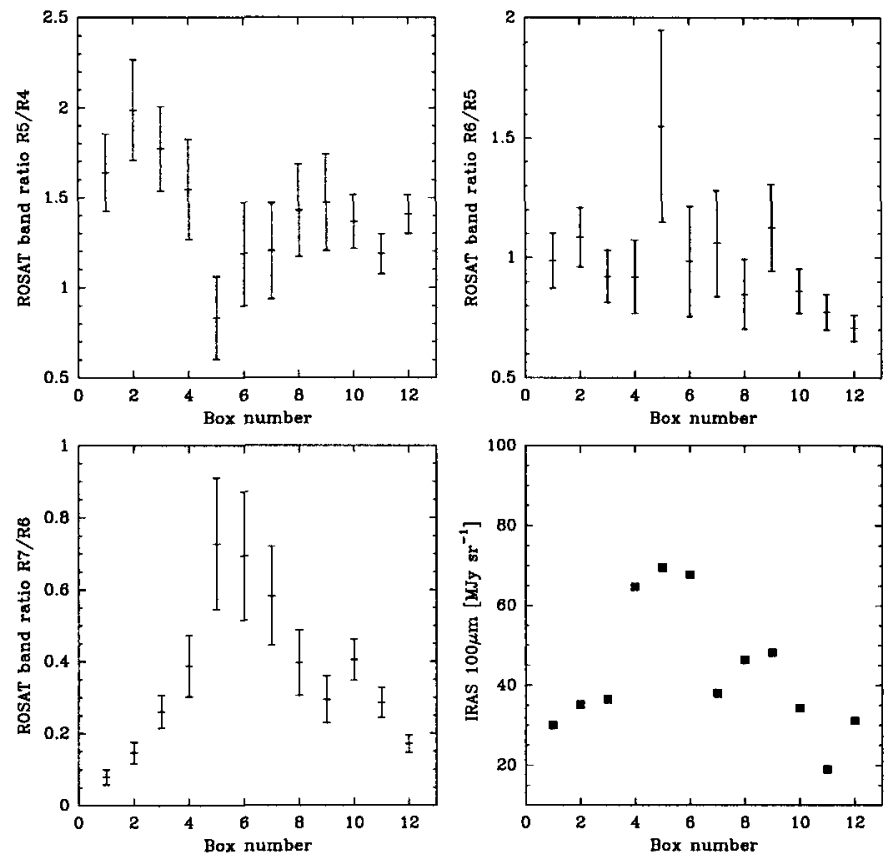

Fig. 1. This figure shows the ROSAT energy band ratios $\frac{\mathrm{R} 5}{\mathrm{R} 4}, \frac{\mathrm{R} 6}{\mathrm{R} 5}$ and $\frac{\mathrm{R} 7}{\mathrm{R} 6}$ across the area of the X-ray shadow described in Blondiau et al. (1997). The boxes 5 to 9 are located at the shadow while the other boxes are located in X-ray bright "OFF-shadow-positions". In the bottom right-hand panel the IRAS $100 \mu \mathrm{m}$ intensity which serves as a tracer for the total X-ray absorbing column density is plotted versus the same box positions. The $\frac{\mathrm{R} 7}{\mathrm{R} 6}$ energy band ratio shows the expected behaviour, because the X-ray spectrum is hardened after absorption. The $\frac{R_{5}}{R_{4}}$ energy band ratio shows the opposite behaviour, which can be explained if one assumes the existence of a foreground diffuse soft X-ray emitting gas.

\section{Energy Band Ratios and Radiation Transfer}

First hints to disclose $\mathrm{X}$-ray shadows are given by a positional anticorrelation of X-ray intensity versus HI column density or IRAS $100 \mu \mathrm{m}$ intensity. The latter serves as a tracer for the total X-ray absorbing column density of neutral and molecular gas. The behaviour of ROSAT band ratios in the regions of X-ray shadows in the LMC was investigated in my thesis (Blondiau 1996). It turned out that in every case the behaviour of the $\mathrm{R} 5 / \mathrm{R} 4$ band ratio across the X-ray shadow is in contrast to the behaviour in the R7/R6 and R6/R5 band ratios. For a definition of the ROSAT energy bands see Snowden et al. (1994). The best explanation for this is the existence of a cooler X-ray plasma in front of the absorbing clouds which gives a significant contribution to the R4 band intensity. Of course, this contribution can be produced by the 


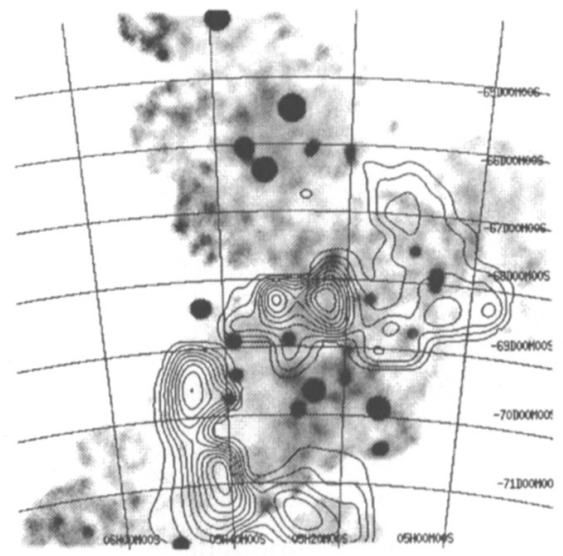

Fig. 2. This image shows a map of the X-ray emission in the $1 / 4 \mathrm{keV}$ band in the region of the LMC. It consists of 172 individual ROSAT pointed observations and is centered at $\operatorname{RA}(2000)=5^{\mathrm{h}} 30^{\mathrm{m}}, \operatorname{DEC}(2000)=-68^{\circ}$. The map covers a sky area of $4^{\circ}$ radius and its angular resolution is about $8^{\prime}$. The linear intensity scale ranges from 5 to $10 \cdot 10^{-4} \mathrm{ctss}^{-1} \operatorname{arcmin}^{-2}$. The contour plot shows the distribution of HI column density in the so-called HI-L-component (Luks \& Rohlfs 1992). The contour lines are in steps of $10 \%$ to $100 \%$ of the maximum value. The maximum column density is about $1.8 \cdot 10^{21} \mathrm{~cm}^{-2}$. The angular resolution of the HI-data is $15^{\prime}$.

galactic halo emission of our own galaxy. Fig. 2 shows the distribution of the $1 / 4 \mathrm{keV}$ diffuse $\mathrm{X}$-ray emission across the whole LMC region which is rather uniform in comparison to the emission in the R4 to $\mathrm{R} 7$ ROSAT energy bands (Blondiau et al. 1996). This is what one would expect if the galactic X-ray emission and the galactic absorption do not much change on angular scales of Fig. $2\left(8^{\prime}\right)$. Therefore one has to solve a radiation transfer equation to decide whether there is an additional LMC contribution in the ROSAT R4 band or not. In case of the largest $\mathrm{X}$-ray shadow I presented a radiation transfer calculation in the ROSAT R4 - R7 bands. I used typical values for galactic halo X-ray intensities (Snowden et al. 1993) and for the cosmological background (Hasinger et al. 1993) intensities and subtracted them from the observed values. Then one has to solve the following equation to determine whether there is LMC X-ray intensity originating in the foreground (FRONT) of the absorbing cloud or not, $I_{\mathrm{X}}=I_{\mathrm{FRONT}}+I_{\mathrm{BACK}} e^{-\sigma_{\mathrm{X}} N_{\mathrm{X}}}$. Here $\mathrm{X}$ denotes both, the ON-shadow and OFF-shadow-position. The absorbing column densities are derived from the $I R A S 100 \mu \mathrm{m}$ emission. Converting these emission values leads to an estimation of the total X-ray absorbing column density on the lines of sight. At the OFF-position one gets $N_{\mathrm{OFF}}=(5.2 \pm 0.5) \cdot 10^{21} \mathrm{~cm}^{-2}$ and on the $\mathrm{ON}$-position $N_{\mathrm{ON}}=(11.1 \pm 0.5) \cdot 10^{21} \mathrm{~cm}^{-2}$. As expected Tab. 1 shows that there is an additional LMC X-ray foreground only in the R4 band present, which accounts for the correct solution of the radiation transfer. So, 
Table 1. Count rates in units of $10^{-6} \mathrm{cts} \mathrm{s}^{-1} \operatorname{arcmin}^{-2}$ in the ROSAT energy bands R4 to R7 ON and OFF the shadow. BACK and FRONT give the count rates from the absorption and after subtraction of the non-LMC components.

\begin{tabular}{ccccc}
\hline$I_{\mathrm{X}}$ & $\mathrm{R} 4$ & $\mathrm{R} 5$ & $\mathrm{R} 6$ & $\mathrm{R} 7$ \\
\hline OFF-shadow & $363 \pm 5$ & $627 \pm 7$ & $586 \pm 7$ & $144 \pm 4$ \\
ON-shadow & $107 \pm 2$ & $127 \pm 2$ & $139 \pm 2$ & $76 \pm 2$ \\
Galactic halo & 37 & 49 & 8 & 0 \\
Cosm. background & 33 & 26 & 72 & 45 \\
BACK & $3500 \pm 700$ & $4600 \pm 900$ & $2800 \pm 400$ & $260 \pm 20$ \\
FRONT & $70 \pm 20$ & $20 \pm 40$ & $-20 \pm 50$ & $-10 \pm 20$ \\
\hline
\end{tabular}

there should be a second softer LMC intrinsic X-ray component in front of the absorbing cloud which also should show up in the ROSAT $1 / 4 \mathrm{keV}$ band.

\section{Interpretation}

As a result of my thesis, it turned out that the HI-L-component is located in front of the $\mathrm{D}$-component, because most $\mathrm{X}$-ray shadows are associated with absorbing HI-clouds of low radial velocities, therefore belonging to the HI-L-component. In the last section I showed that there is some evidence for a second soft X-ray plasma that should show up below the energy of the ROSAT R4 band, that means in the $1 / 4 \mathrm{keV}$ band. Fig. 2 shows the HI column density distribution of the L-component superimposed on the $\mathrm{X}$-ray intensity distribution in the ROSAT $1 / 4 \mathrm{keV}$ band in the LMC area. Right at the eastern wing of the L-component the X-ray intensity is relatively low. In this area the L-component seems to absorb the LMC contribution of the $1 / 4 \mathrm{keV}$ band radiation. So, there are hints for a soft X-ray plasma ("I $I_{\text {FRONT" }}$ ") situated in front of the X-ray absorption clouds which weaken the $T=10^{6.7} \mathrm{~K}$ " $I_{\mathrm{BACK}}$ " X-ray plasma. It is only absorbed by the eastern wing of the HI-L-component, which is the absorbing gas layer of the LMC that is located also in front of the softer X-ray plasma denoted as $I_{\mathrm{FRONT}}$.

\section{References}

Blondiau, M.J. (1996): Ph.D. thesis, University of Bonn

Blondiau, M.J., Kerp, J., Mebold, U., Klein, U. (1996): MPE Report, 263, 321

Blondiau, M.J., Kerp, J., Mebold, U., Klein, U. (1997): A\&A 323, 585

Hasinger, G., et al. (1993): A\&A 275, 1

Luks, Th., Rohlfs, K. (1992): A\&A 263, 41

Snowden, S.L., McCammon, D., Verter, F. (1993): ApJL 409, L21

Snowden, S.L., et al. (1994): ApJ 424, 714

Wang, Q., Hamilton, T., Helfand, D.J., Wu, X. (1991): ApJ 374, 475 